\title{
Indium-Catalyzed Direct Chlorination of Alcohols Using Chlorodimethylsilane-Benzil as a Selective and Mild System
}

Makoto Yasuda , Satoshi Yamasaki, Yoshiyuki Onishi, and Akio Baba*

Department of Molecular Chemistry and Handai Frontier Research Center, Graduate School of Engineering, Osaka University, 2-1 Yamadaoka, Suita, Osaka 565-0871, Japan

\section{Supporting Information}

General. IR spectra were recorded as thin films or as solids in $\mathrm{KBr}$ pellets on a HORIBA FT-720 spectrophotometer. ${ }^{1} \mathrm{H},{ }^{13} \mathrm{C}$ and ${ }^{29} \mathrm{Si} \mathrm{NMR}$ spectra were obtained with a $270,67.9$ and $53.6 \mathrm{MHz}$ spectrometer, respectively, with TMS as internal standard. Mass spectra were recorded on a JEOL JMS-DS303 spectrometer. GLC analyses were performed on a Shimadzu GC-14A with FID using a $15 \mathrm{~m} \times 3 \mathrm{~mm}$ capillary column packed with TC-WAX, TC-5, or TC-1701 $(0.25 \mu \mathrm{m})$. Column chromatography was performed on silica gel (MERK C60). Bulb-to-bulb distillation (Kugelrohr) was accomplished in a Sibata GTO-250RS at the oven temperature and pressure indicated. Yields were determined by GLC or ${ }^{1} \mathrm{H}$ NMR using internal standards.

Materials. Dichloromethane, dehydrated (stabilized with 2-methyl-2-butene) was used without purification. 1,2-Dichloroethane was distilled after the removal of $\mathrm{H}_{2} \mathrm{O}$ by $\mathrm{CaH}_{2}$. All additives in Chart 1, $\mathrm{HSiMe}_{2} \mathrm{Cl}, \mathrm{HSiPh}_{2} \mathrm{Cl}, \mathrm{InCl}_{3}, \mathrm{AlCl}_{3}, \mathrm{Sc}(\mathrm{OTf})_{3}$, alcohols 1a-n, 1p, 1r, and 13 were commercially available. The alcohol 1q was prepared by known method. ${ }^{1}$ The alcohols $\mathbf{1 0}$ and $\mathbf{1 0}$ were prepared as shown below.

Ethyl $p$-(hydroxymethyl)benzoate (10). $p$-(Hydroxymethyl)benzoic acid (7.0 mmol) and $\mathrm{H}_{2} \mathrm{SO}_{4}(3 \mathrm{~mL})$ was added to a stirred solution in EtOH $(30 \mathrm{~mL})$ at reflux. After stirring for $8 \mathrm{~h}$, aqueous $\mathrm{NaHCO}_{3}$ was added to the reaction mixture. The mixture was extracted with ethyl acetate. The organic layer was washed with water and dried over $\mathrm{MgSO}_{4}$. The volatiles were evaporated and the residue was purified by chromatography (ethyl acetate) on silica gel to give the product $(0.79 \mathrm{~g}$, 60\%). bp: $140{ }^{\circ} \mathrm{C} / 0.1 \mathrm{mmHg}$; IR: (neat) $3448(\mathrm{OH}), 1716(\mathrm{C}=\mathrm{O}) \mathrm{cm}^{-1} ;{ }^{1} \mathrm{H}$ NMR $(270 \mathrm{MHz}$, $\left.\mathrm{CDCl}_{3}\right) 8.00(\mathrm{~d}, J=8.3 \mathrm{~Hz}, 2 \mathrm{H}, o), 7.40(\mathrm{~d}, J=8.3 \mathrm{~Hz}, 2 \mathrm{H}, m), 4.74\left(\mathrm{~s}, 2 \mathrm{H}, \mathrm{CH}_{2} \mathrm{OH}\right), 4.36(\mathrm{q}, J=$ $7.1 \mathrm{~Hz}, 2 \mathrm{H}, \mathrm{CH}_{3} \mathrm{CH}_{2}$ ), 2.47 (brs, $\left.1 \mathrm{H}, \mathrm{OH}\right), 1.39$ (t, $\left.J=7.1 \mathrm{~Hz}, 3 \mathrm{H}, \mathrm{CH}_{3}\right) ;{ }^{13} \mathrm{C} \mathrm{NMR}(67.9 \mathrm{MHz}$, $\left.\mathrm{CDCl}_{3}\right) 166.47(\mathrm{C}=\mathrm{O}), 145.87(p), 129.66(o), 129.42(i), 126.31(m), 64.55\left(\mathrm{CH}_{2} \mathrm{OH}\right), 60.98$ 
$\left(\mathrm{CH}_{2}\right), 14.31\left(\mathrm{CH}_{3}\right)$; MS: (EI, $\left.70 \mathrm{eV}\right) \mathrm{m} / z 180\left(\mathrm{M}^{+}, 22\right), 135\left(\mathrm{M}^{+}\right.$- EtO, 100); HRMS: (EI, $\left.70 \mathrm{eV}\right)$ calcd for $\mathrm{C}_{10} \mathrm{H}_{12} \mathrm{O}_{3} 180.0786$ found $\mathrm{m} / z, 180.0775\left(\mathrm{M}^{+}\right)$. Anal. Calcd for $\mathrm{C}_{10} \mathrm{H}_{12} \mathrm{O}_{3}: \mathrm{C}, 66.65 ; \mathrm{H}$, 6.71. Found C, 66.11; H, 6.76.

15-Methylhexadecan-1,15-diol (10). ${ }^{2}$ Methyllithium $(87.5 \mathrm{mmol})$ was added to a stirred solution of oxacyclohexadecan-2-one $(35.0 \mathrm{mmol})$ in dry THF $(60 \mathrm{~mL})$ at $-78{ }^{\circ} \mathrm{C}$. The reaction mixture was stirred at $-78{ }^{\circ} \mathrm{C}$ for $0.5 \mathrm{~h}$ and was allowed to warm to room temperature over $6 \mathrm{~h}$. Acetic acid $(87.5 \mathrm{mmol})$ was added to the reacion mixture and the white precipitate was formed. After stirring for $12 \mathrm{~h}$, the precipitate was filtered off and the filtrate was washed with water, extracted with diethylether and then dried over $\mathrm{MgSO}_{4}$. The volatiles were evaporated to give the crude product as a yellow viscous liquid. purification by recrystalization (9:1 hexane/ EtOAc) gave the pure product as a white solid $(3.1 \mathrm{~g}, 36 \%)$. mp: $62{ }^{\circ} \mathrm{C}$; IR: $(\mathrm{KBr}) 3367(\mathrm{OH}) \mathrm{cm}^{-1} ;{ }^{1} \mathrm{H}$ NMR $\left(600 \mathrm{MHz}, \mathrm{CDCl}_{3}\right) 3.63\left(\mathrm{t}, J=6.7 \mathrm{~Hz}, 2 \mathrm{H}, 1-\mathrm{H}_{2}\right), 1.59$ (brs, 2H, 1-OH and 15-OH, $\mathrm{D}_{2} \mathrm{O}-$ exchangeable), $1.56\left(\mathrm{tt}, J=6.7,6.7 \mathrm{~Hz}, 2 \mathrm{H}, 2-\mathrm{H}_{2}\right), 1.47-1.44\left(\mathrm{~m}, 2 \mathrm{H}, 14-\mathrm{H}_{2}\right), 1.34-1.32(\mathrm{~m}, 4 \mathrm{H}, 3$, $\left.13-\mathrm{H}_{2}\right), 1.34-1.26\left(\mathrm{~m}, 18 \mathrm{H}, 4,5,6,7,8,9,10,11\right.$ and $\left.12-\mathrm{H}_{2}\right), 1.20\left(\mathrm{~s}, 6 \mathrm{H}, 16-\mathrm{H}_{3}, 15-\mathrm{CH}_{3}\right) ;{ }^{13} \mathrm{C}$ NMR (150 MHz, CDCl ${ }_{3}$ ) 71.06 (s, C-15), 62.98 (t, C-1), 43.99 (t, C-14), 32.80 (t, C-2), 30.19 (C12), Some signals appear between 29.70-29.50 ppm as a broad peak, 29.43 (C-4), 29.20 (C-16, 15$\mathrm{CH}_{3}$ ), 25.75 (C-3), 24.37 (C-13); MS: (CI, $\left.70 \mathrm{eV}\right) \mathrm{m} / \mathrm{z} 273\left(\mathrm{M}^{+}+1,3\right), 255\left(\mathrm{M}^{+}+1-\mathrm{H}_{2} \mathrm{O}, 100\right)$; HRMS: (CI, $70 \mathrm{eV}$ ) calcd for $\mathrm{C}_{17} \mathrm{H}_{37} \mathrm{O}_{2} 273.2794$ found $m / z 273.2789\left(\mathrm{M}^{+}+1\right)$. Anal. Calcd for $\mathrm{C}_{17} \mathrm{H}_{36} \mathrm{O}_{2}: \mathrm{C}, 74.94 ; \mathrm{H}, 13.32$. Found: C, 74.80; H, 13.08.

General procedure for chlorination of alcohols (Tables 1 and 2). To a mixture of $\operatorname{InCl}_{3}(0.1$ $\mathrm{mmol})$, benzil $(2.0 \mathrm{mmol})$ and alcohol $1(2.0 \mathrm{mmol})$ in dichloromethane (or dichloroethane in the reaction at $\left.80{ }^{\circ} \mathrm{C}\right)(4.0 \mathrm{~mL})$ was added chlorodimethylsilane $\left(\mathrm{HSiMe}_{2} \mathrm{Cl}\right)(2.2 \mathrm{mmol})$ under nitrogen. The reaction mixture was stirred under the reaction conditions noted in the text. The resulting mixture was poured into aqueous $\mathrm{NaHCO}_{3}(50 \mathrm{~mL})$ and extracted with EtOAc $(50 \mathrm{~mL})$. The organic layer was dried over $\mathrm{MgSO}_{4}$ and concentrated in vacuo. The procedures of further purification for new compounds are shown in the Product data section.

Reaction of 1a using various 1,2-dicarbonyl additives (Chart 1). All reactions were carried out using additives described in Chart 1 instead of bezil under the same reaction conditions of the general procedure.

Reaction of chlorosilyl ether 5 under an $\mathrm{InCl}_{3}$ /benzil system (ref. 7). To a mixture of $\mathrm{InCl}_{3}$ $(0.1 \mathrm{mmol})$ and benzil $(2.0 \mathrm{mmol})$ in dichloromethane $(4.0 \mathrm{~mL})$ was added chlorosilyl ether 5 (2.0 $\mathrm{mmol}$ ). The reaction mixture was stirred at room temperature for $7 \mathrm{~h}$. The resulting mixture was 
poured into aqueous $\mathrm{NaHCO}_{3}(50 \mathrm{~mL})$ and extracted with EtOAc $(50 \mathrm{~mL})$. The organic layer was dried over $\mathrm{MgSO}_{4}$ and concentrated in vacuo. The formation of the chloride 3a (82\% yield) was confirmed by ${ }^{1} \mathrm{H}$ NMR.

Product data. The spectral data of $\mathbf{3 r}$ was in an excellent agreement with the reported data. ${ }^{3}$ The spectral data of $\mathbf{2 a}, \mathbf{3 e}, \mathbf{3 g}, \mathbf{3 j}-\mathbf{m}, \mathbf{3 p},{ }^{4}$ and $\mathbf{1 4}$ were in an excellent agreement with those of commercially available products. Spectral data for the products, 3a-d, 3f, 3h, 3n, 3o, 3q, 11, and 12 are shown below.

2-Chloro-1-phenylpropane (3a). According to the general procedure, this compound was prepared from $\mathrm{HSiMe}_{2} \mathrm{Cl}, \mathbf{1 a}$, benzil and $\mathrm{InCl}_{3}$ in dichloromethane to give the product as a colorless liquid after chromatography (hexane). Further purification was performed by distillation under reduced pressure: bp: $50{ }^{\circ} \mathrm{C} / 0.1 \mathrm{mmHg}$; IR: (neat) 3027, 2973, 2927, 2865 (alkyl) cm ${ }^{-1} ;{ }^{1} \mathrm{H}$ NMR $\left(270 \mathrm{MHz}, \mathrm{CDCl}_{3}\right)$ 7.35-7.20 (m, 5H, aroma), 4.24 (qdd, $\left.J=6.6,7.1,6.8 \mathrm{~Hz}, 1 \mathrm{H}, 2-\mathrm{H}\right), 3.09$ (dd, $J$ $\left.=13.9,6.8 \mathrm{~Hz}, 1 \mathrm{H}, 1-\mathrm{H}^{\mathrm{A}}\right), 2.96\left(\mathrm{dd}, J=13.9,6.8 \mathrm{~Hz}, 1 \mathrm{H}, 1-\mathrm{H}^{\mathrm{B}}\right), 1.51\left(\mathrm{~d}, J=6.6 \mathrm{~Hz}, 3 \mathrm{H}, 3-\mathrm{H}_{3}\right) ;{ }^{1} \mathrm{H}$ NMR (270 MHz, $\mathrm{CD}_{2} \mathrm{Cl}_{2}$ ) 7.35-7.20 (m, 5H, aroma), 4.24 (qdd, $J=6.4,7.1,6.6 \mathrm{~Hz}, 1 \mathrm{H}, 2-\mathrm{H}$ ), $3.05\left(\mathrm{dd}, J=13.9,7.1 \mathrm{~Hz}, 1 \mathrm{H}, 1-\mathrm{H}^{\mathrm{A}}\right), 2.98\left(\mathrm{dd}, J=13.9,6.6 \mathrm{~Hz}, 1 \mathrm{H}, 1-\mathrm{H}^{\mathrm{B}}\right), 1.50(\mathrm{~d}, J=6.4 \mathrm{~Hz}$, $\left.3 \mathrm{H}, 3-\mathrm{H}_{3}\right)$; ${ }^{13} \mathrm{C}$ NMR (67.9 MHz, $\left.\mathrm{CDCl}_{3}\right) 137.90(i), 129.28(\mathrm{~m}), 128.35(o), 126.74(p), 58.52(\mathrm{C}-$ 2), 46.69 (C-1), 24.69 (C-3); ${ }^{13} \mathrm{C}$ NMR (67.9 MHz, $\left.\mathrm{CD}_{2} \mathrm{Cl}_{2}\right) 138.18$ (s, i), 129.35 (d, $m$ ), 128.32 (d, $o$ ), 126.70 (d,p), 58.99 (d, C-2), 46.57 (t, C-1), 24.70 (q, C-3); MS: (EI, $70 \mathrm{eV}) \mathrm{m} / \mathrm{z} 156\left(\mathrm{M}^{+}+2\right.$, 5), $154\left(\mathrm{M}^{+}, 14\right), 91\left(\mathrm{M}^{+}-\mathrm{CH}_{3} \mathrm{CHCl}, 100\right)$; HRMS: (EI, $70 \mathrm{eV}$ ) calcd for $\mathrm{C}_{9} \mathrm{H}_{11} \mathrm{Cl} 154.0549$ found $\mathrm{m} / \mathrm{z}$ 154.0553 $\left(\mathrm{M}^{+}\right)$. Anal. Calcd for $\mathrm{C}_{9} \mathrm{H}_{11} \mathrm{Cl}$ : C, 69.90; H, 7.17; Cl, 22.93. Found: C, 69.61; H, 7.07; Cl, 22.66.

4-Chlorodecane (3b). According to the general procedure, this compound was prepared from $\mathrm{HSiMe}_{2} \mathrm{Cl}, \mathbf{1 b}$, benzil and $\mathrm{InCl}_{3}$ in dichloromethane to give the product as a colorless liquid after distillation under reduced pressure. The obtained fraction includes some rearranged compounds. The main product was in an excellent agreement with the one prepared by known method. ${ }^{5}$ To a solution of thionyl chloride $(4.4 \mathrm{mmol})$ in DMF $(5 \mathrm{~mL})$ was slowly added 4-decanol (4.0 mmol) at $0{ }^{\circ} \mathrm{C}$ and the mixture was heated at $100{ }^{\circ} \mathrm{C}$. After stirring for $1 \mathrm{~h}, \mathrm{H}_{2} \mathrm{O}$ was added to the reaction mixture. The mixture was extracted with diethyl ether. The organic layer was washed with water and dried over $\mathrm{MgSO}_{4}$. The volatiles were evaporated and the residue was purified by chromatography (hexane) on silica gel to give the product. Futher purification was performed by distillation under reduced pressure to give the colorless liquid $(0.224 \mathrm{~g}, 38 \%)$ : bp: $110{ }^{\circ} \mathrm{C} / 30$ mmHg; IR: (neat) 2954, 2938, 2870 (alkyl) $\mathrm{cm}^{-1}$; ${ }^{1} \mathrm{H}$ NMR $\left(270 \mathrm{MHz}, \mathrm{CDCl}_{3}\right) 3.94$ (tt, $J=6.6,6.6$ $\mathrm{Hz}, 1 \mathrm{H}, 4-\mathrm{H}), 1.79-1.64\left(\mathrm{~m}, 4 \mathrm{H}, 3-\mathrm{H}_{2}, 5-\mathrm{H}_{2}\right), 1.64-1.30\left(\mathrm{~m}, 10 \mathrm{H}, 2,6,7,8\right.$ and $\left.9-\mathrm{H}_{2}\right), 0.96(\mathrm{t}, J=$ $\left.7.3 \mathrm{~Hz}, 3 \mathrm{H}, \mathrm{CH}_{3}\right), 0.92\left(\mathrm{t}, J=6.7 \mathrm{~Hz}, 3 \mathrm{H}, \mathrm{CH}_{3}\right) ;{ }^{13} \mathrm{C} \mathrm{NMR}\left(67.9 \mathrm{MHz}, \mathrm{CDCl}_{3}\right) 64.05(\mathrm{~d}, \mathrm{C}-4)$, 
$40.06(\mathrm{t}), 38.57(\mathrm{t}), 31.75(\mathrm{t}), 28.89(\mathrm{t}), 26.49(\mathrm{t}), 22.63(\mathrm{t}), 19.75(\mathrm{t}) 14.11(\mathrm{q}), 13.65(\mathrm{q})$; MS: (EI, $70 \mathrm{eV}) \mathrm{m} / \mathrm{z} 140\left(\mathrm{M}^{+}-\mathrm{HCl}, 37\right), 111\left(\mathrm{M}^{+}-\mathrm{HCl}-\mathrm{C}_{2} \mathrm{H}_{5}, 48\right), 97\left(\mathrm{M}^{+}-\mathrm{HCl}-\mathrm{C}_{3} \mathrm{H}_{7}, 67\right), 55$ (100); Anal. Calcd for $\mathrm{C}_{10} \mathrm{H}_{21} \mathrm{Cl}$ : C, 67.96; H, 11.98; Cl, 20.06. Found: C, 67.90; H, 11.68; Cl, 19.98.

Cyclododecychloride (3c). According to the general procedure, this compound was prepared from $\mathrm{HSiMe}_{2} \mathrm{Cl}, \mathbf{1 c}$, benzil and $\mathrm{InCl}_{3}$ in dichloromethane to give the product as a colorless liquid after distillation under reduced pressure: bp $60{ }^{\circ} \mathrm{C} / 0.11 \mathrm{mmHg} ;{ }^{1} \mathrm{H}$ NMR $(270 \mathrm{MHz}$, $\left.\mathrm{CDCl}_{3}\right) \delta 4.12(1 \mathrm{H}, 1-\mathrm{H}), 1.94\left(2 \mathrm{H}, 2,11-\mathrm{H}^{\mathrm{A}}\right), 1.78\left(2 \mathrm{H}, 2,11-\mathrm{H}^{\mathrm{B}}\right), 1.60-1.22(18 \mathrm{H}) ;{ }^{13} \mathrm{C}$ NMR $\left(67.9 \mathrm{MHz}, \mathrm{CDCl}_{3}\right) \delta 60.35(\mathrm{C}-1), 33.90,23.89,23.46,22.03$; MS (EI, $\left.70 \mathrm{eV}\right) 166\left(\mathrm{M}^{+}-\mathrm{Cl}, 41\right)$. Anal. Calcd for $\mathrm{C}_{12} \mathrm{H}_{23} \mathrm{Cl}$ : C, 70.91; H, 11.38; Cl, 17.48. Found: C, 71.08; H, 11.43; Cl, 17.55.

2-Chloroadamantane (3d). According to the general procedure, this compound was prepared from $\mathrm{HSiMe}_{2} \mathrm{Cl}, \mathbf{1 d}$, benzil and $\mathrm{InCl}_{3}$ in dichloromethane to give the product as a white powder after recrystallization: $\mathrm{mp} 137{ }^{\circ} \mathrm{C} ;{ }^{1} \mathrm{H}$ NMR $\left(270 \mathrm{MHz}, \mathrm{CDCl}_{3}\right) \delta 4.40(\mathrm{~s}, 1 \mathrm{H}, 2-\mathrm{H}), 2.27$ $\left(\mathrm{d}, J=12.70 \mathrm{~Hz}, 2 \mathrm{H}, 4,10-\mathrm{H}^{\mathrm{A}}\right), 2.08(\mathrm{~s}, 2 \mathrm{H}, 1,3-\mathrm{H}), 1.95\left(\mathrm{~d}, J=10.99 \mathrm{~Hz}, 4 \mathrm{H}, 8,9-\mathrm{H}^{\mathrm{B}}\right), 1.84(\mathrm{~d}, J=$ $10.99 \mathrm{~Hz}, 2 \mathrm{H}, 5,7-\mathrm{H}), 1.76(\mathrm{~s}, 2 \mathrm{H}, 6-\mathrm{H}), 1.57$ (d, J= 9.77 Hz, 2H, 4,10-H $\left.{ }^{\mathrm{B}}\right) ;{ }^{13} \mathrm{C}$ NMR $(67.9 \mathrm{MHz}$, $\mathrm{CDCl}_{3}$ ) $\delta 68.28$ (C-2), 38.11 (C-8,9), 37.66 (C-6), 35.76 (C-1,3), 30.95 (C-4,10), 27.40 (C-7), 26.81 (C-5); IR (KBr) $810 \mathrm{~cm}^{-1}$ (C-Cl); MS (EI, $\left.70 \mathrm{eV}\right) 170\left(\mathrm{M}^{+}, 17\right), 134$ (M+ - Cl, 100); HRMS $\left(\mathrm{EI}, 70 \mathrm{eV}\right.$ ) calcd for $\left(\mathrm{C}_{10} \mathrm{H}_{15} \mathrm{Cl}\right) 170.0862\left(\mathrm{M}^{+}\right)$found for $\mathrm{m} / \mathrm{z}$ 170.0849. Anal. Calcd for $\mathrm{C}_{10} \mathrm{H}_{25} \mathrm{Cl}$ : C, 70.37; H, 8.86; Cl, 20.77. Found: C, 70.23; H, 8.50; Cl, 20.86.

2-Chloro-2,5-dimethylhexane (3f). According to the general procedure, this compound was prepared from $\mathrm{HSiMe}{ }_{2} \mathrm{Cl}$, 1f, benzil and $\mathrm{InCl}_{3}$ in dichloromethane to give the product as a colorless liquid after chromatography (pentane). Further purification was performed by distillation under reduced pressure: bp: $50{ }^{\circ} \mathrm{C} / 30 \mathrm{mmHg}$; IR: (neat) 2954, 2870 (alkyl) cm ${ }^{-1}$; ${ }^{1} \mathrm{H}$ NMR (270 $\left.\mathrm{MHz}, \mathrm{CDCl}_{3}\right)$ 1.78-1.70 (m, 2H, 3-H $)$, 1.65-1.46 (m, 1H, 5-H), 1.57 (s, 6H, 1- $\left.\mathrm{H}_{3}, 2-\mathrm{CH}_{3}\right), 1.42-$ $1.31\left(\mathrm{~m}, 2 \mathrm{H}, 4-\mathrm{H}_{2}\right), 0.91\left(\mathrm{~d}, J=6.4 \mathrm{~Hz}, 6 \mathrm{H}, 6-\mathrm{H}_{3}, 5-\mathrm{CH}_{3}\right) ;{ }^{13} \mathrm{C} \mathrm{NMR}\left(67.9 \mathrm{MHz}, \mathrm{CDCl}_{3}\right) 71.25(\mathrm{~s}$, C-2), 44.00 (t, C-3), 34.15 (t, C-4), 32.47 (q, C-1, 2-Me), 28.27 (d, C-5), 22.66 (q, C-6, 5-Me); MS: (EI, $70 \mathrm{eV}) m / z 135\left(\mathrm{M}^{+}+2-\mathrm{Me}\right.$, trace), $133\left(\mathrm{M}^{+}-\mathrm{Me}\right.$, trace), $112\left(\mathrm{M}^{+}-\mathrm{HCl}, 21\right), 77\left(\mathrm{M}^{+}\right.$$\left.\mathrm{C}_{5} \mathrm{H}_{11}, 42\right), 57\left(\mathrm{M}^{+}-\mathrm{C}_{4} \mathrm{H}_{8} \mathrm{Cl}, 100\right)$; Anal. Calcd for $\mathrm{C}_{8} \mathrm{H}_{17} \mathrm{Cl}$ : C, 64.63; H, 11.53; Cl, 23.85. Found: C, 64.52; H, 11.21; Cl, 23.73.

1-Chloroadamantane (3h). According to the general procedure, this compound was prepared from $\mathrm{HSiMe}_{2} \mathrm{Cl}, \mathbf{1 h}$, benzil and $\mathrm{InCl}_{3}$ in dichloromethane to give the product as a white powder after recrystallization: ${ }^{1} \mathrm{H} \mathrm{NMR}\left(270 \mathrm{MHz} \mathrm{CDCl}_{3}\right) \delta 2.11\left(\mathrm{~s}, 9 \mathrm{H}, \mathrm{CH}, \mathrm{CH}_{2}\right), 1.69(\mathrm{~s}, 6 \mathrm{H}$, $\left.\mathrm{CH}_{2}\right) ;{ }^{13} \mathrm{C}$ NMR (67.9 MHz, $\left.\mathrm{CDCl}_{3}\right) \delta 69.01$ (s, C-Cl), 47.96 (s, C-2), $36.75\left(\mathrm{CH}_{2}\right), 31.57\left(\mathrm{CH}_{2}\right)$; MS (EI, $70 \mathrm{eV}) 172\left(\mathrm{M}^{+}+2\right.$, trace), $170\left(\mathrm{M}^{+}, 1\right), 135\left(\mathrm{M}^{+}-\mathrm{Cl}, 100\right)$; HRMS (EI, $\left.70 \mathrm{eV}\right)$ calcd for $\left(\mathrm{C}_{10} \mathrm{H}_{15} \mathrm{Cl}\right) 170.0862\left(\mathrm{M}^{+}\right)$found for $m / z, 170.0854$. 
1-Chloro-1,2-diphenylpropane (3i). ${ }^{6}$ According to the general procedure, this compound was prepared from $\mathrm{HSiMe}_{2} \mathrm{Cl}, \mathbf{1 i}$, benzil and $\mathrm{InCl}_{3}$ in dichloromethane to give the product as a white solid after chromatography (hexane): Mixtures of diastereomers (A:B = ca. 1:1); ${ }^{1} \mathrm{H} \mathrm{NMR}\left(600 \mathrm{MHz}, \mathrm{CDCl}_{3}\right) \delta$ 7.33-7.02 (m, $10 \mathrm{H} \times 2$, Ar from A and B), $4.98(\mathrm{~d}, J=8.3 \mathrm{~Hz}, 1 \mathrm{H}$, 1-H from A), 4.95 (d, $J=8.8 \mathrm{~Hz}, 1 \mathrm{H}, 1-\mathrm{H}$ from B), 3.35 (qd, $J=7.1,8.3 \mathrm{~Hz}, 1 \mathrm{H}, 2-\mathrm{H}$ from B), 3.33 (qd, $J=6.9,8.8 \mathrm{~Hz}, 1 \mathrm{H}, 2-\mathrm{H}$ from A), 1.53 (d, $J=6.9 \mathrm{~Hz}, 3 \mathrm{H}, 3-\mathrm{H}_{3}$ from A) $1.15(\mathrm{~d}, J=7.1$ $\mathrm{Hz}, 3 \mathrm{H}, 3-\mathrm{H}_{3}$ from B); ${ }^{13} \mathrm{C}$ NMR (150 MHz, $\mathrm{CDCl}_{3}$ ) $\delta 143.27$ (s, 2-Ar ipso from B), 142.67 (s, 2Ar ipso from A), 140.63 (s, 1-Ar ipso from A), 140.24 (s, 1-Ar ipso from B), 128.37, 128.26, $128.23,128.15,128.02,127.89,127.87,127.77,127.68,127.52$, 126.88, 126.63, 69.64 (d, C-1 from A), 68.66 (d, C-1 from B), 48.47 (d, C-2 from B), 48.42 (d, C-2 from A), 19.43 (q, C-3 from B), 18.65 (q, C-3 from A); MS (EI, 70 eV) (RT: 12.53 min) $232\left(\mathrm{M}^{+}+2,0.5\right), 230\left(\mathrm{M}^{+}, 1.3\right), 194$ $\left(\mathrm{M}^{+}-\mathrm{HCl}, 8.8\right), 105(100)$ and (RT: $\left.12.94 \mathrm{~min}\right) 232\left(\mathrm{M}^{+}+2,0.6\right), 230\left(\mathrm{M}^{+}, 1.9\right), 194\left(\mathrm{M}^{+}-\mathrm{HCl}\right.$, 5.6), 105 (100); HRMS (EI, $70 \mathrm{eV})$ calcd for $\left(\mathrm{C}_{15} \mathrm{H}_{15} \mathrm{Cl}\right) 230.0862\left(\mathrm{M}^{+}\right)$found for $\mathrm{m} / z, 230.0874$ (RT: $12.53 \mathrm{~min}$ ) and 230.0860 (RT: $12.94 \mathrm{~min})$.

p-Nitrobenzyl chloride (3n). According to the general procedure, this compound was prepared from $\mathrm{HSiMe}_{2} \mathrm{Cl}, \mathbf{1 n}$, benzil and $\mathrm{InCl}_{3}$ in dichloromethane to give the product as a white solid after chromatography (ethyl acetate). Further purification was performed by recrystalization (9:1 hexane/ EtOAc): mp: $72{ }^{\circ} \mathrm{C}$; IR: $(\mathrm{KBr})$ 1535, $1350\left(\mathrm{NO}_{2}\right) \mathrm{cm}^{-1} ;{ }^{1} \mathrm{H}$ NMR $\left(270 \mathrm{MHz}, \mathrm{CDCl}_{3}\right)$ $8.23(\mathrm{~d}, J=8.8 \mathrm{~Hz}, 2 \mathrm{H}, m), 7.57(\mathrm{~d}, J=8.8 \mathrm{~Hz}, 2 \mathrm{H}, o), 4.65\left(\mathrm{~s}, 2 \mathrm{H}, \mathrm{CH}_{2} \mathrm{Cl}\right) ;{ }^{13} \mathrm{C} \mathrm{NMR}(67.9 \mathrm{MHz}$, $\left.\mathrm{CDCl}_{3}\right) 147.78(p), 144.23(\mathrm{i}), 129.23(o), 123.88(\mathrm{~m}), 44.49\left(\mathrm{CH}_{2} \mathrm{Cl}\right)$; MS: (EI, $\left.70 \mathrm{eV}\right) \mathrm{m} / \mathrm{z} 173$ $\left(\mathrm{M}^{+}+\right.$2, 20), $171\left(\mathrm{M}^{+}, 54\right), 136\left(\mathrm{M}^{+}-\mathrm{Cl}, 100\right), 127\left(\mathrm{M}^{+}+2-\mathrm{NO}_{2}, 12\right), 125\left(\mathrm{M}^{+}-\mathrm{NO}_{2}, 34\right)$; HRMS: (EI, $70 \mathrm{eV}$ ) calcd for $\mathrm{C}_{7} \mathrm{H}_{6} \mathrm{ClNO}_{2} 171.0087$ found $\mathrm{m} / z, 171.0069\left(\mathrm{M}^{+}\right)$. Anal. Calcd for $\mathrm{C}_{7} \mathrm{H}_{6} \mathrm{ClNO}_{2}$ : C, 49.00; H, 3.52; Cl, 20.66; N, 8.16. Found: C, 48.76; H, 3.45; Cl, 20.37; N, 8.15.

Ethyl $p$-(chloromethyl)benzoate (3o). According to the general procedure, this compound was prepared from $\mathrm{HSiMe}_{2} \mathrm{Cl}$, 1o, benzil and $\mathrm{InCl}_{3}$ in dichloromethane to give the product as a colorless liquid after chromatography (hexane:ethyl acetate $=99: 1$ ). Further purification was performed by distillation under reduced pressure: bp: $115^{\circ} \mathrm{C} / 0.3 \mathrm{mmHg}$; IR: (neat) $1720(\mathrm{C}=\mathrm{O}) \mathrm{cm}^{-1} ;{ }^{1} \mathrm{H}$ NMR $\left(270 \mathrm{MHz}, \mathrm{CDCl}_{3}\right) 8.04(\mathrm{~d}, J=8.1 \mathrm{~Hz}, 2 \mathrm{H}, o), 7.47(\mathrm{~d}, J=8.1$ $\mathrm{Hz}, 2 \mathrm{H}, m), 4.62\left(\mathrm{~s}, 2 \mathrm{H}, \mathrm{CH}_{2} \mathrm{Cl}\right), 4.39$ (q, $\left.J=7.1 \mathrm{~Hz}, 2 \mathrm{H}, \mathrm{CH}_{3} \mathrm{CH}_{2}\right), 1.40$ (t, $J=7.1 \mathrm{~Hz}, 3 \mathrm{H}, \mathrm{CH}_{3}$ ); ${ }^{13} \mathrm{C} \mathrm{NMR}\left(67.9 \mathrm{MHz}, \mathrm{CDCl}_{3}\right) 166.01(\mathrm{C}=\mathrm{O}), 142.02(p), 130.39(i), 129.91(o), 128.38(m), 61.10$ $\left(\mathrm{CH}_{2}\right), 45.42\left(\mathrm{CH}_{2} \mathrm{Cl}\right), 14.35\left(\mathrm{CH}_{3}\right)$; MS: (EI, $\left.70 \mathrm{eV}\right) \mathrm{m} / \mathrm{z} 200\left(\mathrm{M}^{+}+2,6\right), 198\left(\mathrm{M}^{+}, 22\right), 155\left(\mathrm{M}^{+}\right.$ + 2 - EtO, 31), $153\left(\mathrm{M}^{+}\right.$- EtO, 100); HRMS: (EI, $70 \mathrm{eV}$ ) calcd for $\mathrm{C}_{10} \mathrm{H}_{11} \mathrm{ClO}_{2} 198.0448$ found $m / z$ 198.0444 $\left(\mathrm{M}^{+}\right)$. Anal. Calcd for $\mathrm{C}_{10} \mathrm{H}_{11} \mathrm{ClO}_{2}$ : C, 60.46; H, 5.58; Cl, 17.85. Found: C, 60.32; H, 5.60; Cl, 17.65. 
Ethyl 2-chloro-2-phenylpropanoate (3q). According to the general procedure, this compound was prepared from $\mathrm{HSiMe}_{2} \mathrm{Cl}, \mathbf{1 q}$, benzil and $\mathrm{InCl}_{3}$ in dichloromethane to give the product as a colorless liquid after distillation under reduced pressure: bp $80{ }^{\circ} \mathrm{C} / 0.11 \mathrm{mmHg} ;{ }^{1} \mathrm{H}$ $\operatorname{NMR}\left(270 \mathrm{MHz}, \mathrm{CDCl}_{3}\right) \delta$ 7.43-7.31 (m, 5H, aroma), 5.35 (dd, $\left.J=5.81 \mathrm{~Hz}, 8.91 \mathrm{~Hz}, 1 \mathrm{H}, 3-\mathrm{H}\right)$, $4.16\left(\mathrm{q}, J=6.83 \mathrm{~Hz}, 2 \mathrm{H}, 1^{\prime}\right.$ '-H), $3.17\left(\mathrm{dd}, J=8.91 \mathrm{~Hz}, 15.66 \mathrm{~Hz}, 1 \mathrm{H}, 2-\mathrm{H}^{\mathrm{A}}\right), 3.02(\mathrm{dd}, J=5.81 \mathrm{~Hz}$, $\left.15.66 \mathrm{~Hz}, 3 \mathrm{H}, 2-\mathrm{H}^{\mathrm{B}}\right), 1.23\left(\mathrm{t}, J=6.83 \mathrm{~Hz}, 3 \mathrm{H}, 2^{\prime}-\mathrm{H}\right) ;{ }^{13} \mathrm{C} \mathrm{NMR}\left(67.9 \mathrm{MHz}, \mathrm{CDCl}_{3}\right) \delta 169.52(\mathrm{~s}$, $\mathrm{C}=\mathrm{O}$ ), 140.24 (s, ipso-Ph), 128.75 (d, Ph), 128.68 (d, Ph), 126.89 (d, Ph), 61.00 (t, C-1'), 58.08 (d, C-3), 44.87 (t, C-2), 14.08 (q, C-2'); IR (neat) $1740 \mathrm{~cm}^{-1}$ (C=O); MS (EI, $\left.70 \mathrm{eV}\right) 214\left(\mathrm{M}^{+}+\right.$2, 20), $212\left(\mathrm{M}^{+}, 57\right), 183\left(\mathrm{M}^{+}-\mathrm{C}_{2} \mathrm{H}_{5}, 37\right), 167$ (M+ - OEt, 19), 91 (Bn, 11), 77 (Ph, 29); HRMS (EI, 70 $\mathrm{eV})$ calcd for $\left(\mathrm{C}_{11} \mathrm{H}_{13} \mathrm{O}_{2} \mathrm{Cl}\right) 212.0604$ found for $\mathrm{m} / \mathrm{z} 212.0613\left(\mathrm{M}^{+}\right)$.

Reaction of $1 q$ with $\mathbf{P C l}_{3}$. To a solution of alcohol $\mathbf{1 q}(1.0 \mathrm{mmol})$ in dry dichloromethane $(2 \mathrm{~mL})$ was added $\mathrm{PCl}_{3}(1.0 \mathrm{mmol})$ at $\mathrm{rt}$ for $1 \mathrm{~h}$. Aqueous $\mathrm{NaHCO}_{3}(15 \%, 10 \mathrm{~mL})$ was added to the reaction mixture. The mixture was extracted with ethyl acetate. The organic layer was washed with water and dried over $\mathrm{MgSO}_{4}$. The volatiles were evaporated under reduced pressure, and the residue showed a complicated mixture by NMR mesurement.

Reaction of $1 q$ with $\mathbf{P C l}_{5}$. To a solution of alcohol $\mathbf{1 q}(1.0 \mathrm{mmol})$ in dry $\mathrm{CCl}_{4}(1 \mathrm{~mL})$ was added $\mathrm{PCl}_{5}(1.0 \mathrm{mmol})$ at $77{ }^{\circ} \mathrm{C}$ for $30 \mathrm{~min}$. Aqueous $\mathrm{NaHCO}_{3}(15 \%, 10 \mathrm{~mL})$ was added to the reaction mixture. The mixture was extracted with ethyl acetate. The organic layer was washed with water and dried over $\mathrm{MgSO}_{4}$. The volatiles were evaporated under reduced pressure, and the residue showed the formation of $\mathbf{3 q}(66 \%)$ and ethyl-3-phenyl-2-propenoate $(5 \%)$ by NMR measurement.

Reaction of $1 q$ with $\mathbf{P P h}_{3} / \mathbf{C C l}_{4}$. To a solution of alcohol $\mathbf{1 q}(1.0 \mathrm{mmol})$ in dry $\mathrm{CCl}_{4}(1 \mathrm{~mL})$ was added $\mathrm{PPh}_{3}(1.3 \mathrm{mmol})$ at $77{ }^{\circ} \mathrm{C}$ for $12 \mathrm{~h}$. Aqueous $\mathrm{NaHCO}_{3}(15 \%, 10 \mathrm{~mL})$ was added to the reaction mixture. The mixture was extracted with ethyl acetate. The organic layer was washed with water and dried over $\mathrm{MgSO}_{4}$. The volatiles were evaporated under reduced pressure, and the residue showed the formation of $\mathbf{3 q}(62 \%)$ and ethyl-3-phenyl-2-propenoate (8\%) by NMR measurement.

Methanol mediated procedure for chlorination of alcohol 1r (Tables 2, entry 17). To a mixture of $\mathrm{InCl}_{3}(0.1 \mathrm{mmol})$, benzil $(2.0 \mathrm{mmol})$ and methanol $(2.0 \mathrm{mmol})$ in dichloromethane $(4.0$ $\mathrm{mL})$ was added $\mathrm{HSiMe}_{2} \mathrm{Cl}(2.2 \mathrm{mmol})$ under nitrogen. The reaction mixture was stirred for $1 \mathrm{~h}$ with generating quantitative $\mathrm{H}_{2}$. The addition of the alcohol $1 \mathbf{r}$ and the mixture was stirred for $0.1 \mathrm{~h}$. The resulting mixture was poured into aquous $\mathrm{NaHCO}_{3}(50 \mathrm{~mL})$ and extracted with EtOAc $(50 \mathrm{~mL})$. 
The organic layer was dried over $\mathrm{MgSO}_{4}$ and concentrated in vacuo. The spectral data of $\mathbf{3 r}$ was in an excellent agreement with the reported data. ${ }^{3}$

\section{Selective Chlorination of Unsymmetrical Diol 10.}

$\mathrm{Me}_{2} \mathrm{SiHCl} / \mathrm{benzil} / \mathrm{InCl}_{3}$ System; 15-Chloro-15-methylhexadecan-1-ol (11). To a mixture of $\mathrm{InCl}_{3}(0.1 \mathrm{mmol})$, benzil $(2.0 \mathrm{mmol})$ and alcohol $10(2.0 \mathrm{mmol})$ in dichloromethane $(4.0$ $\mathrm{mL})$ was added $\mathrm{HSiMe}_{2} \mathrm{Cl}(2.2 \mathrm{mmol})$ under nitrogen. The reaction mixture was stirred at room temperature for $12 \mathrm{~h}$. The resulting mixture was poured into EtOAc $(50 \mathrm{~mL})$ and washed by aqueous $\mathrm{NaHCO}_{3}(50 \mathrm{~mL})$. The organic layer was dried over $\mathrm{MgSO}_{4}$ and concentrated in vacuo. The volatiles were evaporated and the residue was purified by chromatography (ethyl acetate) on silica gel to give the product 11. Futher purification was performed by distillation under reduced pressure to give the colorless liquid, which turned to a white solid after $1 \mathrm{~h}$ : bp: $150{ }^{\circ} \mathrm{C} / 0.1 \mathrm{mmHg}$; mp: $36^{\circ} \mathrm{C}$; IR: (KBr) $3367(\mathrm{OH}) \mathrm{cm}^{-1}$; ${ }^{1} \mathrm{H} \mathrm{NMR}\left(600 \mathrm{MHz}, \mathrm{CDCl}_{3}\right) 3.53\left(\mathrm{t}, J=6.8 \mathrm{~Hz}, 2 \mathrm{H}, 1-\mathrm{H}_{2}\right)$, 2.78 (brs, 1H, OH, $\mathrm{D}_{2} \mathrm{O}$-exchangeable), 1.67-1.63 (m, 2H, 14- $\left.\mathrm{H}_{2}\right), 1.52-1.45\left(\mathrm{~m}, 2 \mathrm{H}, 2-\mathrm{H}_{2}\right), 1.49$ (s, $\left.6 \mathrm{H}, 16-\mathrm{H}_{3}, 15-\mathrm{CH}_{3}\right), 1.41-1.38\left(\mathrm{~m}, 2 \mathrm{H}, 13-\mathrm{H}_{2}\right), 1.24-1.20(\mathrm{~m}, 20 \mathrm{H}, 3,4,5,6,7,8,9,10,11$ and $12-$ $\mathrm{H}_{2}$ ); ${ }^{13} \mathrm{C}$ NMR (150 MHz, $\mathrm{CDCl}_{3}$ ) 71.06 (s, C-15), 62.61 (t, C-1), 45.98 (t, C-14), 32.57 (t, C-2), 32.27 (t, C-16), Some signals appear between 29.62-29.36 ppm as a broad peak, 25.66 (t, C-3), 24.99 (t, C-13); MS: (EI, $70 \mathrm{eV}) \mathrm{m} / z 254\left(\mathrm{M}^{+}-\mathrm{HCl}, 3\right), 236\left(\mathrm{M}^{+}-\mathrm{HCl}-\mathrm{H}_{2} \mathrm{O}, 6\right), 69$ (100); HRMS: (CI, $70 \mathrm{eV}$ ) calcd for $\mathrm{C}_{17} \mathrm{H}_{35} \mathrm{O} 255.2688$ found $\mathrm{m} / z, 255.2675\left(\mathrm{M}^{+}+1-\mathrm{HCl}\right)$. Anal. Calcd for $\mathrm{C}_{17} \mathrm{H}_{35} \mathrm{ClO}: \mathrm{C}, 70.19 ; \mathrm{H}, 12.13 ; \mathrm{Cl}, 12.19$. Found: C, 70.31; H, 11.98; Cl, 12.27.

$\mathbf{P P h}_{3} / \mathbf{C C l}_{4}$ System; 16-Chloro-2-methylhexadecan-2-ol (12). To a solution of alcohol $10(1.0 \mathrm{mmol})$ in dry $\mathrm{CCl}_{4}(1 \mathrm{~mL})$ was added $\mathrm{PPh}_{3}(1.3 \mathrm{mmol})$ at $77{ }^{\circ} \mathrm{C}$ for $12 \mathrm{~h}$. Aqueous $\mathrm{NaHCO}_{3}(15 \%, 10 \mathrm{~mL})$ was added to the reaction mixture. The mixture was extracted with ethyl acetate. The organic layer was washed with water and dried over $\mathrm{MgSO}_{4}$. The volatiles were evaporated and the residue was purified by distillation under reduced pressure to give the product 12 as a colorless liquid: bp: $160{ }^{\circ} \mathrm{C} / 0.1 \mathrm{mmHg}$; IR: (neat) $3371(\mathrm{OH}) \mathrm{cm}^{-1}$; ${ }^{1} \mathrm{H} \mathrm{NMR}(600 \mathrm{MHz}$, $\left.\mathrm{CDCl}_{3}\right) 3.53\left(\mathrm{t}, J=6.8 \mathrm{~Hz}, 2 \mathrm{H}, 16-\mathrm{H}_{2}\right), 1.76\left(\mathrm{tt}, J=7.4,6.8 \mathrm{~Hz}, 2 \mathrm{H}, 15-\mathrm{H}_{2}\right), 1.48-1.46(\mathrm{brs}, 1 \mathrm{H}$, $\mathrm{OH}, \mathrm{D}_{2} \mathrm{O}$-exchangeable), 1.47-1.45 (m, 2H, 3- $\left.\mathrm{H}_{2}\right), 1.42\left(\mathrm{tt}, J=7.4,7.4 \mathrm{~Hz}, 2 \mathrm{H}, 14-\mathrm{H}_{2}\right) 1.35-1.33$ $\left(\mathrm{m}, 2 \mathrm{H}, 4-\mathrm{H}_{2}\right), 1.29-1.25\left(\mathrm{~m}, 18 \mathrm{H}, 5,6,7,8,9,10,11,12\right.$ and $\left.13-\mathrm{H}_{2}\right), 1.20\left(\mathrm{~s}, 6 \mathrm{H}, 1-\mathrm{H}_{3}, 2-\mathrm{CH}_{3}\right) ;{ }^{13} \mathrm{C}$ NMR (150 MHz, CDCl $\left.{ }_{3}\right) 71.06$ (s, C-2), 45.23 (t, C-16), 44.01 (t, C-14), 32.67 (t, C-15), 30.23 (C5), Some signals appear between 29.64-29.49 ppm as a broad peak, $29.24\left(\mathrm{C}-1,2-\mathrm{CH}_{3}\right), 28.92(\mathrm{C}-$ 13), 26.91 (t, C-14), 24.39 (t, C-4); MS: (EI, 70 eV) m/z $277\left(\mathrm{M}^{+}+2\right.$ - Me, 5), $275\left(\mathrm{M}^{+}-\mathrm{Me}, 14\right)$, $274\left(\mathrm{M}^{+}+2-\mathrm{H}_{2} \mathrm{O}, 0.7\right), 272\left(\mathrm{M}^{+}-\mathrm{H}_{2} \mathrm{O}, 2\right), 59$ (100); HRMS: (EI, $70 \mathrm{eV}$ ) calcd for $\mathrm{C}_{17} \mathrm{H}_{33} \mathrm{Cl}$ 
272.2271 found $m / z$ 272.2289 $\left(\mathrm{M}^{+}-\mathrm{H}_{2} \mathrm{O}\right)$. Anal. Calcd for $\mathrm{C}_{17} \mathrm{H}_{35} \mathrm{ClO}: \mathrm{C}, 70.19 ; \mathrm{H}, 12.13$; $\mathrm{Cl}$, 12.19; O, 5.50. Found: C, 69.93; H, 11.83; Cl, 11.95 .

$\mathbf{P C l}_{5}$ System. To a solution of alcohol $\mathbf{1 0}(1.0 \mathrm{mmol})$ in dry $\mathrm{CCl}_{4}(1 \mathrm{~mL})$ was added $\mathrm{PCl}_{5}$ $(0.5 \mathrm{mmol})$ at $77{ }^{\circ} \mathrm{C}$ for $12 \mathrm{~h}$. Aqueous $\mathrm{NaHCO}_{3}(15 \%, 10 \mathrm{~mL})$ was added to the reaction mixture. The mixture was extracted with ethyl acetate. The organic layer was washed with water and dried over $\mathrm{MgSO}_{4}$. The volatiles were evaporated under reduced pressure, and the residue showed the formation of $12(45 \%)$.

\section{Competitive Chlorination between 1 and 13.}

$\mathrm{Me}_{2} \mathrm{SiHCl} / \mathrm{benzil} / \mathrm{InCl}_{3}$ System. To a mixture of $\mathrm{InCl}_{3}(0.1 \mathrm{mmol})$, benzil $(2.0 \mathrm{mmol})$ and alcohols 1 f $(2.0 \mathrm{mmol})$ and $\mathbf{1 3}(2.0 \mathrm{mmol})$ in dichloromethane $(4.0 \mathrm{~mL})$ was added $\mathrm{HSiMe}_{2} \mathrm{Cl}(2.2$ mmol) under nitrogen. The reaction mixture was stirred at room temperature for $12 \mathrm{~h}$. The resulting mixture was poured into diethyl ether and washed by aqueous $\mathrm{NaHCO}_{3}(50 \mathrm{~mL})$. The organic layer was dried over $\mathrm{MgSO}_{4}$ and concentrated in vacuo. The volatiles were evaporated under reduced pressure, and the residue showed formation of $\mathbf{3 f}(90 \%)$ and recovery of $\mathbf{1 3}$ (93\%) by NMR measurement.

$\mathbf{P P h}_{3} / \mathbf{C C l}_{4}$ System. To a solution of alcohols $\mathbf{1 f}(1.0 \mathrm{mmol})$ and $\mathbf{1 3}(1.0 \mathrm{mmol})$ in dry $\mathrm{CCl}_{4}(1 \mathrm{~mL})$ was added $\mathrm{PPh}_{3}(1.3 \mathrm{mmol})$ at $77{ }^{\circ} \mathrm{C}$ for $1 \mathrm{~h}$. Aqueous $\mathrm{NaHCO}_{3}(15 \%, 10 \mathrm{~mL})$ was added to the reaction mixture. The mixture was extracted with diethyl ether. The organic layer was washed with water and dried over $\mathrm{MgSO}_{4}$. The volatiles were evaporated under reduced pressure, and the residue showed the formation of $\mathbf{1 4}(85 \%)$ and recovery of $\mathbf{1 f}(92 \%)$ by NMR measurement.

$\mathbf{P C l}_{5}$ System. To a solution of alcohols $\mathbf{1 f}(1.0 \mathrm{mmol})$ and $\mathbf{1 3}(1.0 \mathrm{mmol})$ in dry $\mathrm{CCl}_{4}(1$ $\mathrm{mL})$ was added $\mathrm{PCl}_{5}(0.5 \mathrm{mmol})$ at $77{ }^{\circ} \mathrm{C}$ for $30 \mathrm{~min}$. Aqueous $\mathrm{NaHCO}_{3}(15 \%, 10 \mathrm{~mL})$ was added to the reaction mixture. The mixture was extracted with diethyl ether. The organic layer was washed with water and dried over $\mathrm{MgSO}_{4}$. The volatiles were evaporated under reduced pressure, and the residue showed the formation of $\mathbf{1 4}(73 \%)$ and $\mathbf{3 f}(15 \%)$. The recovery of $\mathbf{1 f}$ (ca. $80 \%$ ) was observed.

\section{Control Experiment (Scheme 1).}

Uncatalyzed Reaction of 1a with $\mathrm{HSiMe}_{2} \mathrm{Cl}$. To a reaction mixture of alcohol 1a (2 mmol) in dichloromethane $(4 \mathrm{~mL})$ was added $\mathrm{HSiMe}_{2} \mathrm{Cl}(2.2 \mathrm{mmol})$ at room temperature for $1 \mathrm{~h}$. Removal of the volatiles gave a crude mixture. The formation of silyl ether 4 (ca. 30\%) was 
confirmed by ${ }^{1} \mathrm{H}$ NMR $\left(\mathrm{CD}_{2} \mathrm{Cl}_{2}\right)$. The signals were in an excellent agreement with those of purely isolated authentic sample, that was prepared as follows (Chart A and Chart B).

1-Phenyl-2-dimethylsiloxypropane (4). To a mixture of ammonium chloride $(0.07 \mathrm{~g}$, $1.3 \mathrm{mmol})$ and 1-phenyl-2-propanol $(5.54 \mathrm{~g}, 41 \mathrm{mmol})$ in a single neck round bottom flask was added 1,1,3,3-tetramethyldisilazane $(5.12 \mathrm{~g}, 38 \mathrm{mmol})$. The mixture was stirred in room temperature for $20 \mathrm{~h}$. Removal of the ammonia and unreacted disilazane by evaporation gave a crude mixture. The resultant mixture was distilled to give the product as a colorless liquid $(3.21 \mathrm{~g}, 40 \%): \mathrm{bp}: 75^{\circ} \mathrm{C}$ / $0.1 \mathrm{mmHg}$; IR: (neat) 2114 (Si-H), 906 (Si-H) cm ${ }^{-1} ;{ }^{1} \mathrm{H} \mathrm{NMR} \mathrm{(270} \mathrm{MHz,} \mathrm{CDCl}_{3}$ ) 7.31-7.17 (m, $5 \mathrm{H}$, aroma), $4.84\left(\mathrm{sep}, J=2.9 \mathrm{~Hz}, 1 \mathrm{H}, \mathrm{Si}-\mathrm{H}\right.$ satellite signals observed $\left.{ }^{1} J_{\mathrm{Si}-\mathrm{H}}=200.9 \mathrm{~Hz}\right), 3.97$ (qdd, $J=6.1,7.1,5.9 \mathrm{~Hz}, 1 \mathrm{H}, 2-\mathrm{H}), 2.75\left(\mathrm{dd}, J=13.2,7.1 \mathrm{~Hz}, 1 \mathrm{H}, 1-\mathrm{H}^{\mathrm{A}}\right), 2.67(\mathrm{dd}, J=13.2,5.9 \mathrm{~Hz}, 1 \mathrm{H}$, $\left.1-\mathrm{H}^{\mathrm{B}}\right), 1.10\left(\mathrm{~d}, J=6.1 \mathrm{~Hz}, 3 \mathrm{H}, 3-\mathrm{H}_{3}\right), 0.103\left(\mathrm{~d}, J=2.9 \mathrm{~Hz}, 3 \mathrm{H}, \mathrm{Si}_{-} \mathrm{CH}_{3}{ }^{\mathrm{A}}\right), 0.014(\mathrm{~d}, J=2.9 \mathrm{~Hz}, 3 \mathrm{H}$, $\mathrm{Si}-\mathrm{CH}_{3}{ }^{\mathrm{B}}$ ); ${ }^{1} \mathrm{H} \mathrm{NMR}\left(270 \mathrm{MHz}, \mathrm{CD}_{2} \mathrm{Cl}_{2}\right)$ 7.30-7.17 (m, 5H, aroma), 4.49 (sep, J=2.9 Hz, 1H, Si-H, satellite signals observed $\left.{ }^{1} J_{\mathrm{Si}-\mathrm{H}}=200.4 \mathrm{~Hz}\right), 3.98(\mathrm{qdd}, J=6.1,6.8,5.6 \mathrm{~Hz}, 1 \mathrm{H}, 2-\mathrm{H}), 2.75(\mathrm{dd}, J=$ 13.2, $\left.6.8 \mathrm{~Hz}, 1 \mathrm{H}, 1-\mathrm{H}^{\mathrm{A}}\right), 2.67\left(\mathrm{dd}, J=13.2,5.6 \mathrm{~Hz}, 1 \mathrm{H}, 1-\mathrm{H}^{\mathrm{B}}\right), 1.17\left(\mathrm{~d}, J=6.1 \mathrm{~Hz}, 3 \mathrm{H}, 3-\mathrm{H}_{3}\right), 0.092$ $\left(\mathrm{d}, J=2.9 \mathrm{~Hz}, 3 \mathrm{H}, \mathrm{Si}-\mathrm{CH}_{3}{ }^{\mathrm{A}}\right), 0.015\left(\mathrm{~d}, J=2.9 \mathrm{~Hz}, 3 \mathrm{H}, \mathrm{Si}-\mathrm{CH}_{3}{ }^{\mathrm{B}}\right) ;{ }^{13} \mathrm{C} \mathrm{NMR}\left(67.9 \mathrm{MHz}, \mathrm{CDCl}_{3}\right)$ $139.06(i), 129.49(m), 128.04(o), 126.01(p), 71.49$ (C-2), 45.92 (C-1), 23.22 (C-3), -1.07 (Si-C $\left.{ }^{\mathrm{A}}\right)$, -1.36 (Si-C ${ }^{\mathrm{B}}$ ); MS: (EI, $\left.70 \mathrm{eV}\right) \mathrm{m} / z, 194\left(\mathrm{M}^{+}, 3\right), 103\left(\mathrm{M}^{+}-\mathrm{PhCH}_{2}, 100\right) ;{ }^{13} \mathrm{C} \mathrm{NMR}(67.9 \mathrm{MHz}$, $\left.\mathrm{CD}_{2} \mathrm{Cl}_{2}\right) 139.36(\mathrm{~s}, i), 129.64(\mathrm{~d}, m), 128.06(\mathrm{~d}, o), 126.03(\mathrm{~d}, p), 71.40$ (d, C-2), 45.90 (t, C-1), 23.13 (q, C-3), -1.23 (q, Si-C ${ }^{\mathrm{A}}$ ), -1.48 (q, Si-C $\mathrm{C}^{\mathrm{B}}$ ); HRMS: (EI, $70 \mathrm{eV}$ ) calcd for $\mathrm{C}_{11} \mathrm{H}_{18} \mathrm{OSi}$ 194.1127 found $\mathrm{m} / \mathrm{z}$ 194.1120 $\left(\mathrm{M}^{+}\right)$. Anal. Calcd for $\mathrm{C}_{11} \mathrm{H}_{18} \mathrm{OSi}$ : C, 67.98; H, 9.34. Found: C, 67.69; H, 9.27.

Catalyzed Reaction of 1a with $\mathrm{HSiMe}_{2} \mathrm{Cl}$. To a mixture of $\mathrm{InCl}_{3}(0.025 \mathrm{mmol})$ and benzil $(0.5 \mathrm{mmol})$ in $\mathrm{CD}_{2} \mathrm{Cl}_{2}(0.75 \mathrm{~mL})$ in NMR tube was added the alcohol $\mathbf{1 a}(0.5 \mathrm{mmol})$ and $\mathrm{HSiMe}_{2} \mathrm{Cl}(0.65 \mathrm{mmol})$. After $2 \mathrm{~h}$, the signal corresponding to silyl ether 5 appeared. The signals were in an excellent agreement with those of purely isolated authentic sample, that was prepared as follows (Chart C and Chart D). Further stirring for $18 \mathrm{~h}$, the signals of chloride $\mathbf{3}$ were observed (Chart E and Chart F).

1-Phenyl-2-(chlorodimethylsiloxy)propane (5). To a solution of 1-phenyl-2-propanol $(8.2 \mathrm{~g}, 60 \mathrm{mmol})$ and triethylamine $(9.1 \mathrm{~g}, 90 \mathrm{mmol})$ in benzene $(35 \mathrm{~mL})$ was slowly added a solution of $\mathrm{Me}_{2} \mathrm{SiCl}_{2}(11.6 \mathrm{~g}, 90 \mathrm{mmol})$ in benzene $(65 \mathrm{~mL})$ at ambient temperature for $1 \mathrm{~h}$. The mixture was stirred at $60{ }^{\circ} \mathrm{C}$ for $3 \mathrm{~h}$. Removal of the resultant salt by the centrifugal separation and evaporation of solvents gave a crude mixture. The resultant mixture was distilled to give the pure product as a colorless liquid (6.2 g, 45\%): bp: $65{ }^{\circ} \mathrm{C} / 0.15 \mathrm{mmHg}$; IR: (neat) 3028, 2974, 2912 (alkyl) $\mathrm{cm}^{-1} ;{ }^{1} \mathrm{H}$ NMR (270 MHz, $\mathrm{CDCl}_{3}$ ) 7.32-7.17 (m, 5H, aroma), 4.19 (qdd, $J=6.1,7.3,5.6$ $\mathrm{Hz}, 1 \mathrm{H}, 2-\mathrm{H}), 2.80\left(\mathrm{~d}, J=13.4,7.3 \mathrm{~Hz}, 1 \mathrm{H}, 1-\mathrm{H}^{\mathrm{A}}\right), 2.71\left(\mathrm{dd}, J=13.4,5.6 \mathrm{~Hz}, 1 \mathrm{H}, 1-\mathrm{H}^{\mathrm{B}}\right), 1.25(\mathrm{~d}, J$ 
$\left.=6.1 \mathrm{~Hz}, 3 \mathrm{H}, 3-\mathrm{H}_{3}\right), 0.39\left(\mathrm{~s}, 3 \mathrm{H}, \mathrm{Si}_{-} \mathrm{CH}_{3}{ }^{\mathrm{A}}\right), 0.13\left(\mathrm{~s}, 3 \mathrm{H}, \mathrm{Si}-\mathrm{CH}_{3}{ }^{\mathrm{B}}\right) ;{ }^{1} \mathrm{H}$ NMR $\left(270 \mathrm{MHz}, \mathrm{CD}_{2} \mathrm{Cl}_{2}\right)$ 7.32-7.18 (m, 5H, aroma), 4.20 (qdd, $J=6.1,7.1,5.6 \mathrm{~Hz}, 1 \mathrm{H}, 2-\mathrm{H}), 2.79(\mathrm{~d}, J=13.2,7.1 \mathrm{~Hz}, 1 \mathrm{H}$, $\left.1-\mathrm{H}^{\mathrm{A}}\right), 2.72\left(\mathrm{dd}, J=13.2,5.6 \mathrm{~Hz}, 1 \mathrm{H}, 1-\mathrm{H}^{\mathrm{B}}\right), 1.24\left(\mathrm{~d}, J=6.1 \mathrm{~Hz}, 3 \mathrm{H}, 3-\mathrm{H}_{3}\right), 0.39\left(\mathrm{~s}, 3 \mathrm{H}, \mathrm{Si}_{-} \mathrm{CH}_{3}{ }^{\mathrm{A}}\right)$, 0.15 (s, 3H, Si-CH$\left.{ }_{3}{ }^{\mathrm{B}}\right) ;{ }^{13} \mathrm{C} \mathrm{NMR}\left(67.9 \mathrm{MHz} \mathrm{CDCl}_{3}\right) 138.72(\mathrm{i}), 129.58(\mathrm{~m}), 128.14(\mathrm{o}), 126.20$ (p), 71.29 (C-2), 45.65 (C-1), $23.12(\mathrm{C}-3), 2.59\left(\mathrm{Si}^{-} \mathrm{C}^{\mathrm{A}}\right), 1.82\left(\mathrm{Si}_{-} \mathrm{C}^{\mathrm{B}}\right) ;{ }^{13} \mathrm{C}$ NMR $(67.9 \mathrm{MHz}$, $\left.\mathrm{CD}_{2} \mathrm{Cl}_{2}\right) 138.94(\mathrm{~s}, i), 129.73(\mathrm{~d}, m), 128.19(\mathrm{~d}, o) 126.25(\mathrm{~d}, p), 71.37$ (d, C-2), 45.63 (t, C-1), 23.04 (q, C-3), $2.41\left(\mathrm{Si}_{-} \mathrm{C}^{\mathrm{A}}\right), 1.64\left(\mathrm{Si}^{\mathrm{B}} \mathrm{C}^{\mathrm{B}}\right)$; MS: (EI, $\left.70 \mathrm{eV}\right) m / z, 230\left(\mathrm{M}^{+}+\right.$2, 0.7), $228\left(\mathrm{M}^{+,} 2.0\right)$, $139\left(\mathrm{M}^{+}+2-\mathrm{PhCH}_{2}, 35\right), 137\left(\mathrm{M}^{+}-\mathrm{PhCH}_{2}, 100\right)$; HRMS: (EI, $\left.70 \mathrm{eV}\right)$ calcd for $\mathrm{C}_{11} \mathrm{H}_{17} \mathrm{ClOSi}$ 228.0737 found $m / z 228.0745\left(\mathrm{M}^{+}\right)$.

\section{References}

1) Yasuda, M.; Onishi, Y.; Ueba, M.; Miyai, T.; Baba, A. J. Org. Chem. 2001, 66, 7741-7744.

2) Hernández, A. S.; Hodges, J. C. J. Org. Chem. 1997, 62, 3153-3157.

3) Strazzolini, P.; Giumanini, A. G.; Verardo, G. Tetrahedron 1994, 50, 217-254.

4) Holcomb, H. L.; Nakanishi, S.; Flood, T. C. Organometallics 1996, 15, 4228-4234.

5) Hudson, R. H.; Spinoza, G. R. J. Chem. Soc., Perkin Trans. 1 1976, 104-108.

6) Kingsbury, C. A.; Best D. C. J. Org. Chem. 1967, 32, 6-10. 
Chart A. Authentic sample $4\left(\mathrm{CD}_{2} \mathrm{Cl}_{2}\right)$
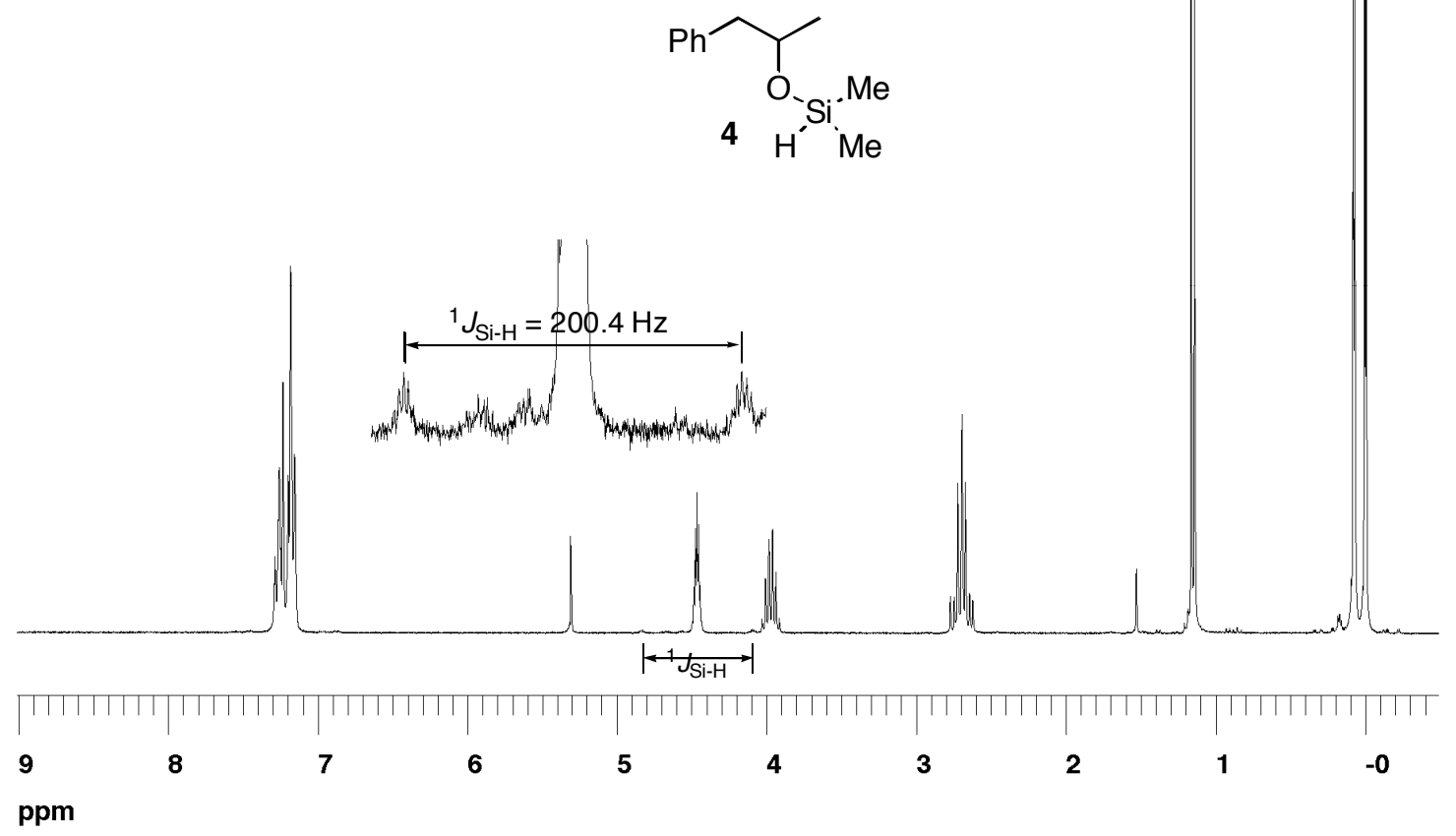

Chart B. Uncatalyzed reaction of 1 a with $\mathrm{HSiMe}_{2} \mathrm{Cl}\left(\mathrm{CD}_{2} \mathrm{Cl}_{2}\right)$
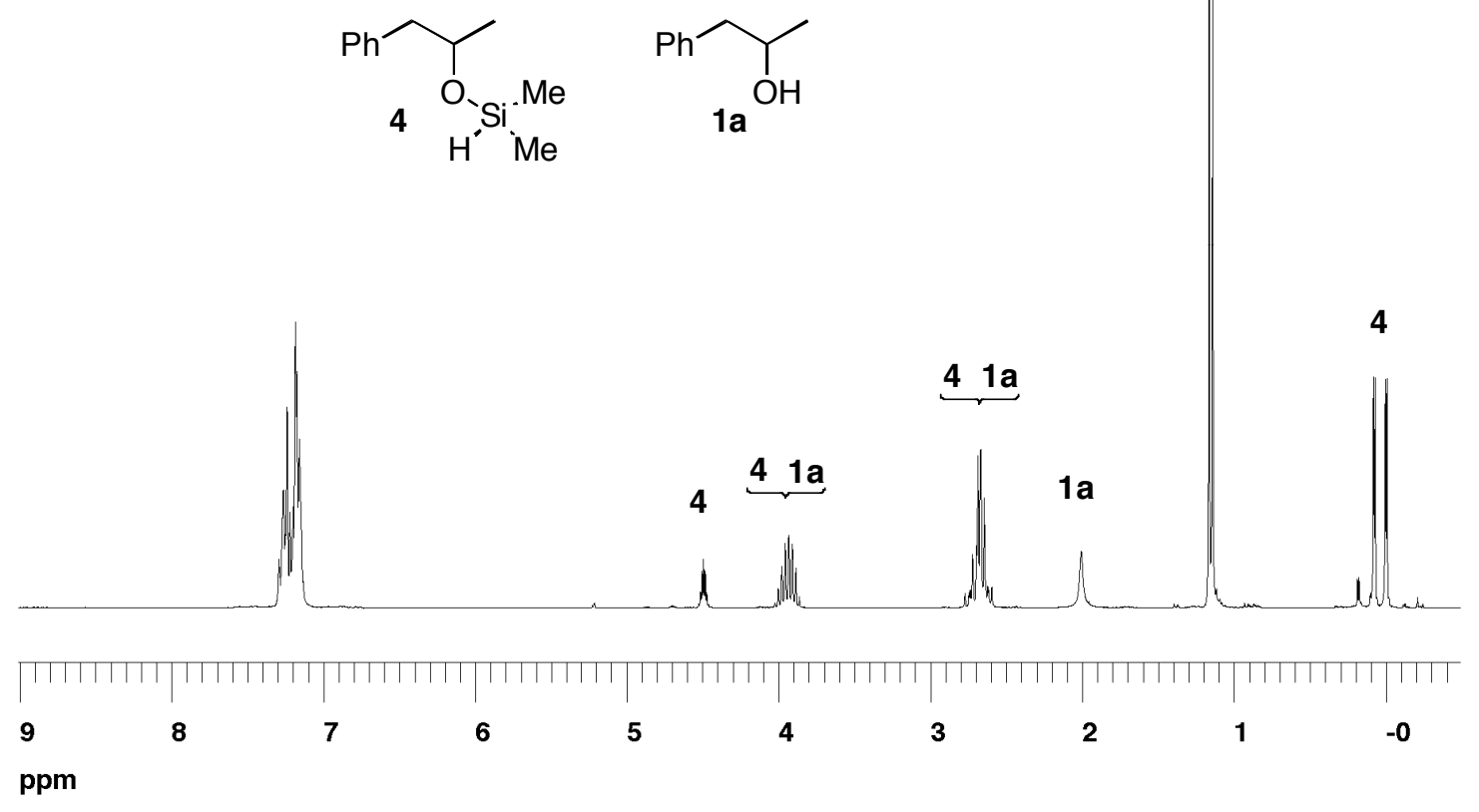
Chart C. Authentic sample $5\left(\mathrm{CD}_{2} \mathrm{Cl}_{2}\right)$

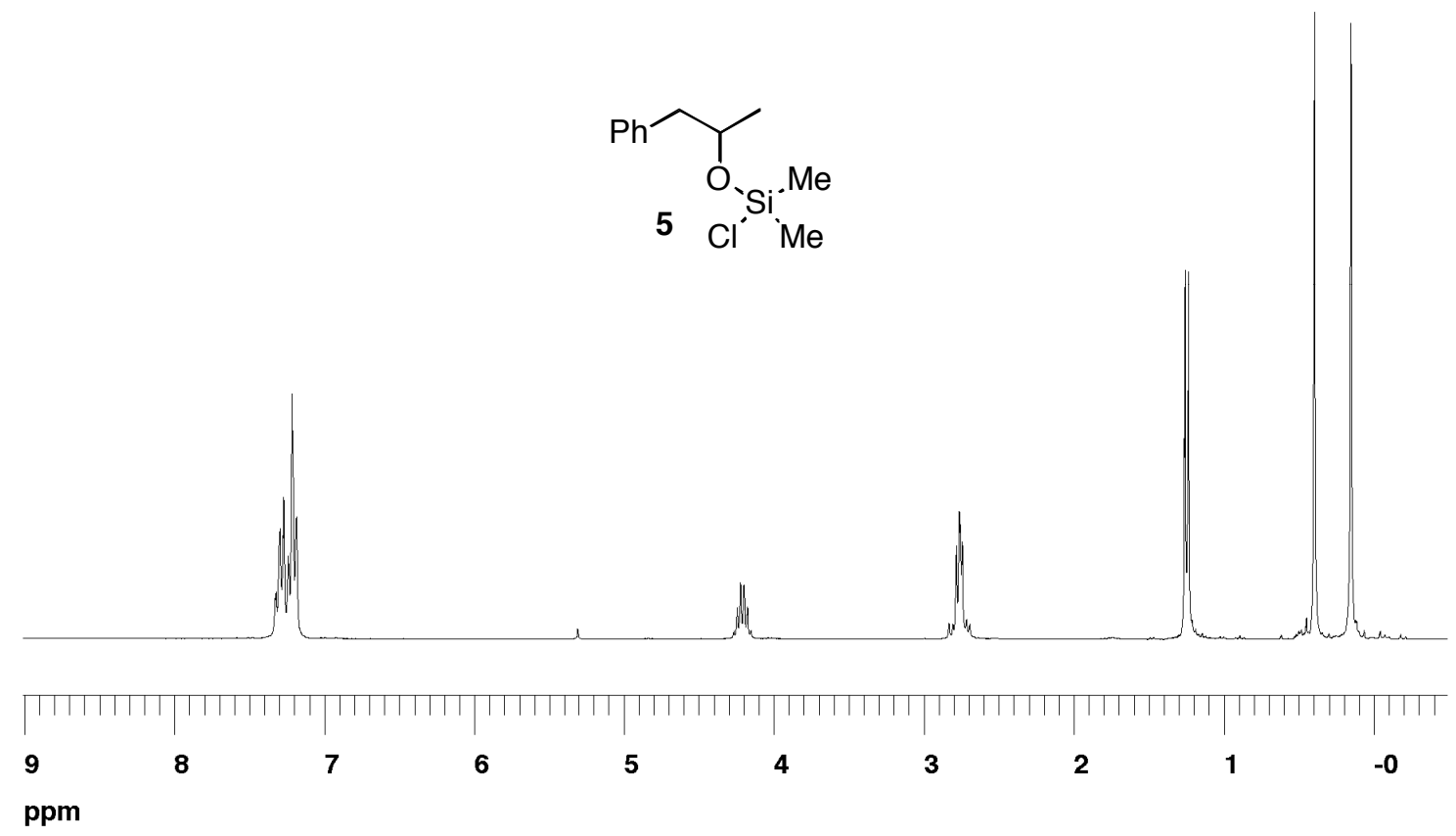

Chart D. Catalyzed reaction of 1 a with $\mathrm{HSiMe}_{2} \mathrm{Cl}$ after $2 \mathrm{~h}\left(\mathrm{CD}_{2} \mathrm{Cl}_{2}\right)$

55

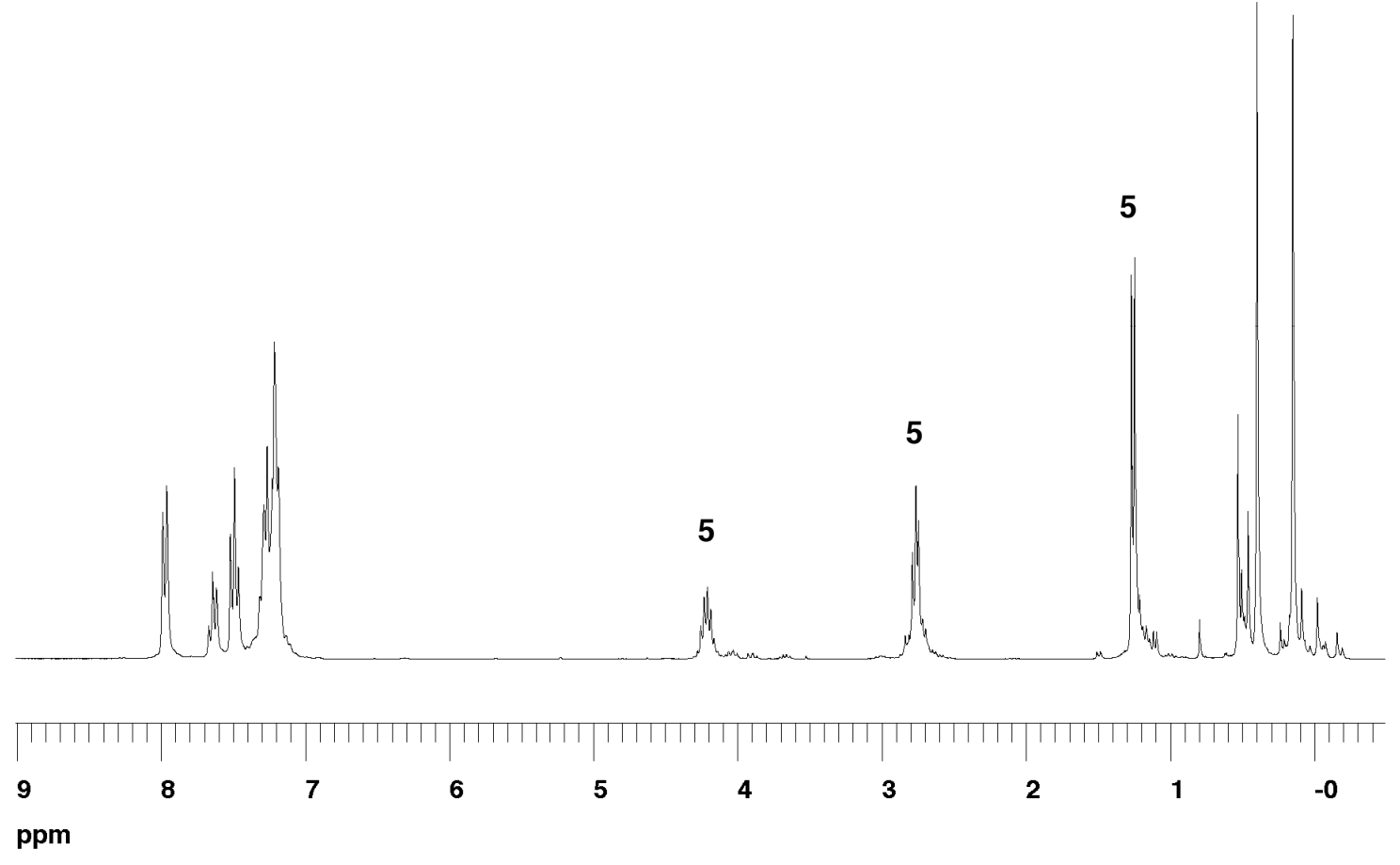


Chart E. Authentic sample $3 a\left(\mathrm{CD}_{2} \mathrm{Cl}_{2}\right)$

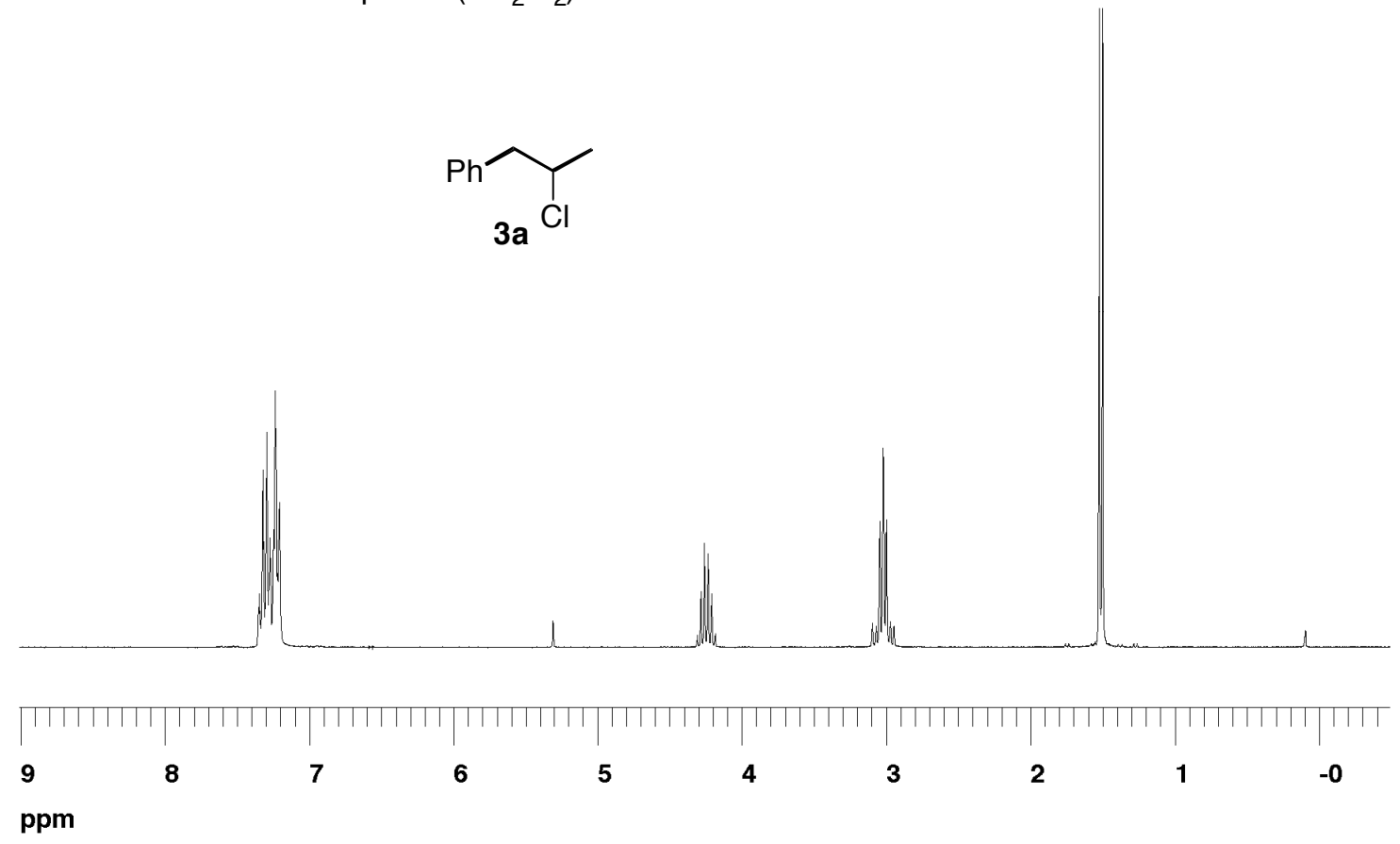

Chart F. Catalyzed reaction of $1 \mathrm{a}$ with $\mathrm{HSiMe}_{2} \mathrm{Cl}$ after $20 \mathrm{~h}\left(\mathrm{CD}_{2} \mathrm{Cl}_{2}\right)$

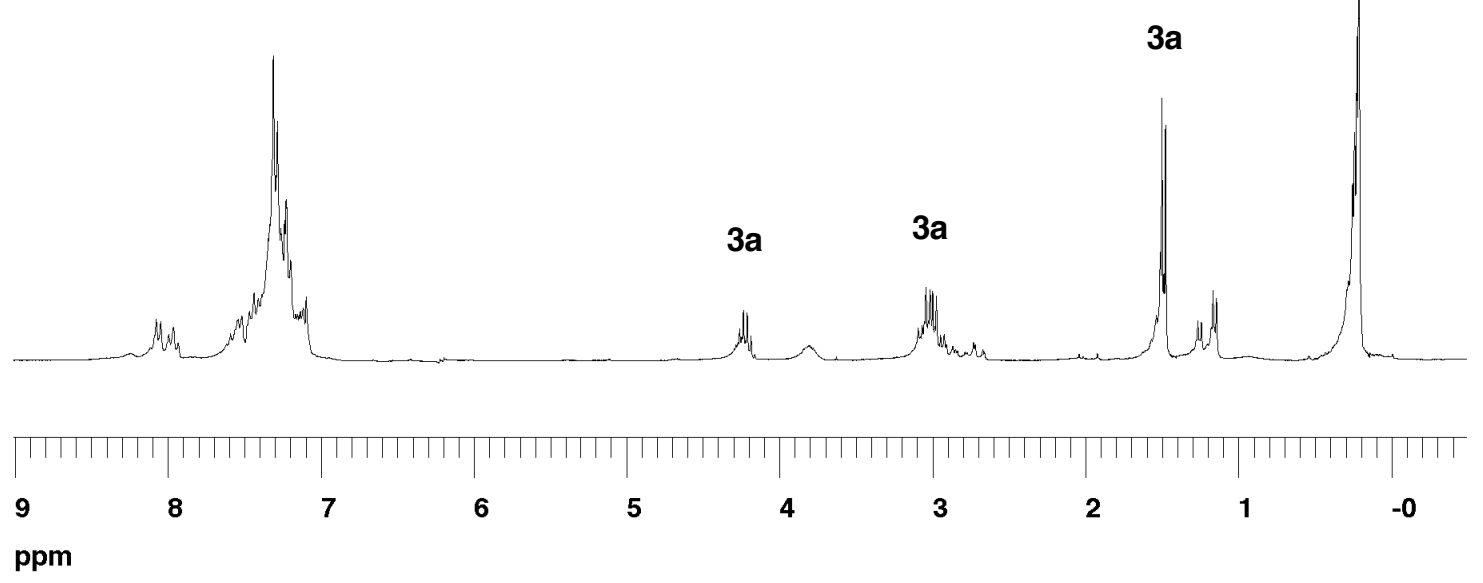

